\title{
Asymptotics of Entropy Rate in Special Families of Hidden Markov Chains
}

\author{
Guangyue Han, Member, IEEE, and Brian H. Marcus, Fellow, IEEE
}

\begin{abstract}
We derive an asymptotic formula for entropy rate of a hidden Markov chain under certain parameterizations. We also discuss applications of the asymptotic formula to the asymptotic behaviors of entropy rate of hidden Markov chains as outputs of certain channels, such as binary symmetric channel, binary erasure channel, and some special Gilbert-Elliot channel.
\end{abstract}

Index Terms-Entropy, entropy rate, hidden Markov chain, hidden Markov model, hidden Markov process.

\section{INTRODUCTION}

C ONSIDER a discrete finite-valued stationary stochastic process $Y=\left\{Y_{n}, n \in \mathbb{Z}\right\}$. The entropy rate of $Y$ is defined to be

$$
H(Y)=\lim _{n \rightarrow \infty} H\left(Y_{-n}^{0}\right) /(n+1)
$$

here, $H\left(Y_{-n}^{0}\right)$ denotes the joint entropy of $Y_{-n}^{0}:=$ $\left\{Y_{-n}, Y_{-n+1}, \ldots, Y_{0}\right\}$, and $\log$ is taken to mean the natural logarithm.

If $Y$ is a Markov chain with alphabet $\mathcal{B}:=\{1,2, \ldots, B\}$ and transition probability matrix $\Delta$, it is well known that $H(Y)$ can be explicitly expressed with the stationary vector of $Y$ and $\Delta$. Let $\mathcal{A}$ denote a finite alphabet $\{1,2, \ldots, A\}$, and let $\Phi$ denote a function defined on alphabet $\mathcal{B}$, taking values in $\mathcal{A}$; then the stochastic process defined by $Z:=\Phi(Y)=\left\{\Phi\left(Y_{n}\right), n \in \mathbb{Z}\right\}$ is called a hidden Markov chain; alternatively a hidden Markov chain can be defined as a Markov chain observed in noise. For a hidden Markov chain $Z, H(Z)$ turns out to be the integral of certain function defined on a simplex with respect to a measure due to Blackwell [4]. However, Blackwell's measure is somewhat complicated and the integral formula appears to be difficult to evaluate in most cases. In general, it is very difficult to compute $H(Z)$; so far there is no simple and explicit formula for $H(Z)$.

Recently, the problem of computing entropy rate of a hidden Markov chain has drawn much interest, and many approaches have been adopted to tackle this problem. For instance, Blackwell's measure has been used to bound the entropy rate [16]

Manuscript received October 13, 2008; revised October 30, 2009. Current version published March 10, 2010. The work of G. Han was supported by the University of Hong Kong by Grant No. 200709159007, and by the Research Grants Council of the Hong Kong Special Administrative Region, China, by Grant No. HKU 701708P.

G. Han is with the Department of Mathematics, The University of Hong Kong, PokFuLam Road, Hong Kong (e-mail: ghan@maths.hku.hk).

B. Marcus is with the Department of Mathematics, The University of British Columbia, Vancouver, B.C., Canada V6T 1Z2 (e-mail: marcus@math.ubc.ca).

Communicated by Y. Kontoyiannis, Associate Editor for Shannon Theory.

Digital Object Identifier 10.1109/TIT.2009.2039094 and a variation on the Birch bound [3] was introduced in [5] An efficient Monte Carlo method for computing the entropy rate of a hidden Markov chain was proposed independently by Arnold and Loeliger [1], Pfister et. al. [18], and Sharma and Singh [20]. The connection between the entropy rate of a hidden Markov chain and the top Lyapunov exponent of a random matrix product has been observed [6], [11]-[13]. In [7], it is shown that under mild positivity assumptions the entropy rate of a hidden Markov chain varies analytically as a function of the underlying Markov chain parameters.

Another recent approach is based on computing the coefficients of an asymptotic expansion of the entropy rate around certain values of the Markov and channel parameters. The first result along these lines was presented in [13], where for a binary symmetric channel with crossover probability $\varepsilon$ [denoted by BSC $(\varepsilon)$ ], the Taylor expansion of $H(Z)$ around $\varepsilon=0$ is studied for a binary hidden Markov chain of order one. In particular, the first derivative of $H(Z)$ at $\varepsilon=0$ is expressed very compactly as a Kullback-Liebler divergence between two distributions on binary triplets, derived from the marginal of the input process $X$. Further improvements and new methods for the asymptotic expansion approach were obtained in [17], [21], [22], and [8]. In [17], the authors express the entropy rate for a binary hidden Markov chain where one of the transition probabilities is equal to zero as an asymptotic expansion including a $O(\varepsilon \log \varepsilon)$ term.

This paper is organized as follows. In Section II we give an asymptotic formula (Theorem 2.8) for entropy rate of a hidden Markov chain around a weak Black Hole. The coefficients in the formula can be computed in principle (although explicit computations may be quite complicated in general). The formula can be viewed as a generalization of that under the Black Hole condition considered in [8]. The weak Black Hole case is important for hidden Markov chains obtained as output processes of noisy channels, corresponding to input processes, for which certain sequences have probability zero. Examples are given in Section III. Example 3.1 was already treated in [9] and [10] for only the first few coefficients; but in this case, these coefficients were computed quite explicitly.

\section{ASYMPTOTIC FORMULA FOR ENTROPY RATE}

Let $W$ be the simplex, comprising the vectors

$$
\left\{w=\left(w_{1}, w_{2}, \ldots, w_{B}\right) \in \mathbb{R}^{B}: w_{i} \geq 0, \sum_{i} w_{i}=1\right\}
$$

and let $W_{a}$ be all $w \in W$ with $w_{i}=0$ for $\Phi(i) \neq a$. For $a \in \mathcal{A}$, let $\Delta_{a}$ denote the $B \times B$ matrix such that $\Delta_{a}(i, j)=\Delta(i, j)$ for $j$ with $\Phi(j)=a$, and $\Delta_{a}(i, j)=0$ otherwise. For $a \in A$, 
define the scalar-valued and vector-valued functions $r_{a}$ and $f_{a}$ on $W$ by

$$
r_{a}(w)=w \Delta_{a} \mathbf{1} \quad \text { and } \quad f_{a}(w)=w \Delta_{a} / r_{a}(w) .
$$

Note that $f_{a}$ defines the action of the matrix $\Delta_{a}$ on the simplex $W$.

Definition 2.1: (see [8]) Suppose that for every $a \in \mathcal{A}, \Delta_{a}$ is a rank one matrix, and every column of $\Delta_{a}$ is either strictly positive or all zeros. We call this the Black Hole case.

Remark 2.2: The term Black Hole comes from the fact that in this case each $f_{a}$ is defined on the whole simplex $W$ and the image of $f_{a}$ on $W$ is a single point.

It was shown [8] that $H(Z)$ is analytic around a Black Hole and the derivatives of $H(Z)$ can be exactly computed around a Black Hole. In this sequel, we consider weakened assumptions and prove an asymptotic formula for entropy rate of a hidden Markov chain around a "weak Black Hole," which contains the Black Hole as a special case, thus, generalizing the corresponding result in [8].

Definition 2.3: Suppose that for every $a \in \mathcal{A}, \Delta_{a}$ is either an all zero matrix or a rank one matrix. We call this the weak Black Hole case.

Let $\Omega$ denote a subset of $\mathbb{R}$ and assume $0 \in \Omega$. For a real function $f(\varepsilon)$ defined on $\Omega$, we say $f(\varepsilon)$ is analytic around $\varepsilon=$ 0 if $f(\varepsilon)$ can be analytically continued to a neighborhood of $\varepsilon=$ 0 in $\mathbb{C}$, thus admitting a Taylor series expansion around $\varepsilon=0$, which converges to $f(\varepsilon)$ around $\varepsilon=0$. For a given analytic function $f(\varepsilon)$ around $\varepsilon=0$, let ord $(f(\varepsilon))$ denote its order, i.e., the degree of the first nonzero term of its Taylor series expansion around $\varepsilon=0$. We say the transition probability matrix $\Delta(\varepsilon)$ is normally parameterized (for concrete examples, see Section III) by $\varepsilon(\varepsilon \geq 0)$ around $\varepsilon=0$ if

1. each entry of $\Delta(\varepsilon)$ is an analytic function around $\varepsilon=0$,

2. when $\varepsilon>0, \Delta(\varepsilon)$ is nonnegative and irreducible,

3. $\Delta(0)$ is a weak black hole, i.e., for every $a \in \mathcal{A}, \Delta_{a}(0)$ is either an all zero matrix or a rank one matrix.

In the following, expressions like $p_{X}(x)$ will be used to mean $P(X=x)$ and we drop the subscripts if the context is clear: $p(x), p(z)$ mean $P(X=x), P(Z=z)$, respectively, and further $p(y \mid x), p\left(z_{0} \mid z_{-n}^{-1}\right)$ mean $P(Y=y \mid X=x)$, $P\left(Z_{0}=z_{0} \mid Z_{-n}^{-1}=z_{-n}^{-1}\right)$, respectively.

Proposition 2.4: Suppose that $\Delta(\varepsilon)$ is analytically parameterized by $\varepsilon \geq 0$ and when $\varepsilon>0, \Delta(\varepsilon)$ is nonnegative and irreducible. Then for any fixed hidden Markov sequence $z_{-n}^{0} \in \mathcal{A}^{n+1}$,

1. $p\left(z_{-n}^{-1}\right)$ is analytic around $\varepsilon=0$;

2. $p\left(y_{i}=\cdot \mid z_{-n}^{i}\right):=\left(p\left(y_{i}=b \mid z_{-n}^{i}\right): b=1,2, \ldots, B\right)$ is analytic around $\varepsilon=0$, where $\cdot$ denotes $B$ possible states of Markov chain $Y$,

3. $p\left(z_{0} \mid z_{-n}^{-1}\right)$ is analytic around $\varepsilon=0$.

Proof: We first prove that when $\varepsilon>0, \Delta(\varepsilon)$ has a unique positive stationary vector $\pi(\varepsilon)$, which can be extended to an analytic function around $\varepsilon=0$.
When $\varepsilon>0, \Delta(\varepsilon)$ is nonnegative and irreducible. By PerronFrobenius theory [19], $\Delta(\varepsilon)$ has a unique positive stationary vector, say $\pi(\varepsilon)$. Since

$$
\operatorname{adj}(I-\Delta(\varepsilon))(I-\Delta(\varepsilon))=\operatorname{det}(I-\Delta(\varepsilon)) I=0
$$

(here $\operatorname{adj}(\cdot)$ denotes the adjugate operator on matrices), one can choose $\pi(\varepsilon)$ to be any normalized row vector of adj $(I-\Delta(\varepsilon))$. So $\pi(\varepsilon)$ can be written as

$$
\frac{\left(\pi_{1}(\varepsilon), \pi_{2}(\varepsilon), \ldots, \pi_{B}(\varepsilon)\right)}{\pi_{1}(\varepsilon)+\pi_{2}(\varepsilon)+\cdots+\pi_{B}(\varepsilon)}
$$

where each $\pi_{i}(\varepsilon)$ is a nonnegative analytic function of $\varepsilon$ and the first nonzero term of Taylor series expansion of each $\pi_{i}(\varepsilon)$ has a positive coefficient. Then we conclude that for each $i$

$$
\operatorname{ord}\left(\pi_{i}(\varepsilon)\right) \geq \operatorname{ord}\left(\pi_{1}(\varepsilon)+\cdots+\pi_{B}(\varepsilon)\right)
$$

and thus $\pi(\varepsilon)$, which is uniquely defined on $\varepsilon>0$, can be continuously extended to $\varepsilon=0$ via setting $\pi(0)=\lim _{\varepsilon \rightarrow 0} \pi(\varepsilon)$ and the extended function is analytic around $\varepsilon=0$.

1. Now

$$
\begin{aligned}
p\left(z_{-n}^{-1}\right) & =\pi(\varepsilon) \Delta_{z_{-n}} \cdots \Delta_{z_{-1}} 1 \\
& =\frac{\left(\pi_{1}(\varepsilon), \pi_{2}(\varepsilon), \ldots, \pi_{B}(\varepsilon)\right) \Delta_{z_{-n}} \cdots \Delta_{z_{-1}} \mathbf{1}}{\pi_{1}(\varepsilon)+\pi_{2}(\varepsilon)+\cdots+\pi_{B}(\varepsilon)} \\
& =: \frac{f(\varepsilon)}{g(\varepsilon)}
\end{aligned}
$$

here ord $(f(\varepsilon)) \geq \operatorname{ord}(g(\varepsilon))$. It then follows that $p\left(z_{-n}^{-1}\right)$ is analytic around $\varepsilon=0$.

2. Let $x_{i,-n}=x_{i,-n}\left(z_{-n}^{i}\right)$ denote $p\left(y_{i}=\cdot \mid z_{-n}^{i}\right)$. Then one checks that $x_{i,-n}$ satisfies the following iteration:

$$
x_{i,-n}=\frac{x_{i-1,-n} \Delta_{z_{i}}}{x_{i-1,-n} \Delta_{z_{i}} \mathbf{1}}, \quad-n \leq i \leq-1
$$

starting with $x_{-n-1,-n}=p\left(y_{-n-1}=\cdot\right)$. Because $\Delta$ is analytically parameterized by $\varepsilon(\varepsilon \geq 0)$ and $\Delta(\varepsilon)$ is nonnegative and irreducible when $\varepsilon>0$, inductively we can prove (the proof is similar to the proof of analyticity of $\pi(\varepsilon)$ ) that for any $i, x_{i,-n}$ can be written as follows:

$$
x_{i,-n}=\frac{\left(f_{1}(\varepsilon), f_{2}(\varepsilon), \ldots, f_{B}(\varepsilon)\right)}{f_{1}(\varepsilon)+f_{2}(\varepsilon)+\cdots+f_{B}(\varepsilon)}
$$

where $f_{i}(\varepsilon)$ 's are analytic functions around $\varepsilon=0$. Note that for each $i$

$$
\operatorname{ord}\left(f_{i}(\varepsilon)\right) \geq \operatorname{ord}\left(f_{1}(\varepsilon)+f_{2}(\varepsilon)+\cdots+f_{B}(\varepsilon)\right) .
$$

The existence of the Taylor series expansion of $x_{i,-n}$ around $\varepsilon=0$ (for any $i$ ) then follows.

3. One checks that

$$
p\left(z_{0} \mid z_{-n}^{-1}\right)=x_{-1,-n} \Delta_{z_{0}} 1 .
$$

Analyticity of $p\left(z_{0} \mid z_{-n}^{-1}\right)$ immediately follows from (3) and analyticity of $x_{-1,-n}$ around $\varepsilon=0$, which has been shown in 2. 
Lemma 2.5: Consider two formal series expansion $f(x), g(x) \in \mathbb{R}[[x]]$ such that $f(x)=\sum_{i=0}^{\infty} f_{i} x^{i}$ and $g(x)=\sum_{i=0}^{\infty} g_{i} x^{i}$, where $g_{0} \neq 0$. Let $h(x) \in \mathbb{R}[[x]]$ be the quotient of $f(x)$ and $g(x)$ with $h(x)=\sum_{i=0}^{\infty} h_{i} x^{i}$. Then $h_{i}$ is a function dependent only on $f_{0}, \ldots, f_{i}$ and $g_{0}, \ldots, g_{i}$.

Proof: Comparing the coefficients of all the terms in the following identity:

$$
\left(\sum_{i=0}^{\infty} h_{i} x^{i}\right)\left(\sum_{i=0}^{\infty} g_{i} x^{i}\right)=\sum_{i=0}^{\infty} f_{i} x^{i}
$$

we obtain that for any $i$,

$$
h_{0} g_{i}+h_{1} g_{i-1}+\cdots+h_{i} g_{0}=f_{i} .
$$

The lemma then follows from an induction (on $i$ ) argument.

For a mapping $v=v(\varepsilon):[0, \infty) \rightarrow W$ analytic around $\varepsilon=0$ and a hidden Markov sequence $z_{-n}^{0}$, define

$$
p_{v}\left(z_{-n}^{-1}\right)=v \Delta_{z_{-n}} \cdots \Delta_{z_{-1}} 1, \quad p_{v}\left(z_{0} \mid z_{-n}^{-1}\right)=\frac{p_{v}\left(z_{-n}^{0}\right)}{p_{v}\left(z_{-n}^{-1}\right)}
$$

for all $i$, define

$$
p_{v}\left(y_{i}=\cdot \mid z_{-n}^{i}\right)=\frac{v \Delta_{z_{-n}} \cdots \Delta_{z_{i}}}{v \Delta_{z_{-n}} \cdots \Delta_{z_{i}}}
$$

where - denotes the possible states of Markov chain $Y$. Let $b_{v, j}\left(z_{-n}^{0}\right)$ denote the coefficient of $\varepsilon^{j}$ in the Taylor series expansion of $p_{v}\left(z_{0} \mid z_{-n}^{-1}\right)$ (note that $b_{v, j}\left(z_{-n}^{0}\right)$ does not depend on $\varepsilon$ )

$$
p_{v}\left(z_{0} \mid z_{-n}^{-1}\right)=\sum_{j=0}^{\infty} b_{v, j}\left(z_{-n}^{0}\right) \varepsilon^{j} .
$$

We have the following lemma.

Lemma 2.6: For two mappings $v=v(\varepsilon)$, $\hat{v}=\hat{v}(\varepsilon):[0, \infty) \rightarrow W$ analytic around $\varepsilon=0$, if ord $\left(p_{v}\left(z_{-n}^{-1}\right)\right)$, ord $\left(p_{\hat{v}}\left(z_{-n}^{-1}\right)\right) \leq k$, we then have

$$
b_{v, j}\left(z_{-n}^{0}\right)=b_{\hat{v}, j}\left(z_{-n}^{0}\right), \quad 0 \leq j \leq n-4 k-1 .
$$

Proof: Let $x_{v, i}=x_{v, i}\left(z_{-n}^{i}\right)=p_{v}\left(y_{i}=\cdot \mid z_{-n}^{i}\right)$ and $x_{\hat{v}, i}=x_{\hat{v}, i}\left(z_{-n}^{i}\right)=p_{\hat{v}}\left(y_{i}=\cdot \mid z_{-n}^{i}\right)$, here again $\cdot$ denotes the possible states of Markov chain $Y$. Consider the Taylor series expansion of $x_{v, i}, x_{\hat{v}, i}$ around $\varepsilon=0$

$$
\begin{aligned}
& x_{v, i}=a_{v, 0}\left(z_{-n}^{i}\right)+a_{v, 1}\left(z_{-n}^{i}\right) \varepsilon+a_{v, 2}\left(z_{-n}^{i}\right) \varepsilon^{2}+\cdots \\
& x_{\hat{v}, i}=a_{\hat{v}, 0}\left(z_{-n}^{i}\right)+a_{\hat{v}, 1}\left(z_{-n}^{i}\right) \varepsilon+a_{\hat{v}, 2}\left(z_{-n}^{i}\right) \varepsilon^{2}+\cdots .
\end{aligned}
$$

We shall show that $a_{v, j}\left(z_{-n}^{i}\right)=a_{\hat{v}, j}\left(z_{-n}^{i}\right)$ for $j$ with

$$
0 \leq j \leq n+i-\sum_{l=-n}^{i} \max \left\{J_{v}\left(z_{-n}^{l}\right), J_{\hat{v}}\left(z_{-n}^{l}\right)\right\},
$$

where for any hidden Markov sequence $z_{-n}^{i}$

$$
\begin{aligned}
& J_{v}\left(z_{-n}^{i}\right)= \\
& \begin{cases}1+\operatorname{ord}\left(p_{v}\left(z_{i} \mid z_{-n}^{i-1}\right)\right) & \text { if ord }\left(p_{v}\left(z_{i} \mid z_{-n}^{i-1}\right)\right)>0 \\
0 & \text { if ord }\left(p_{v}\left(z_{i} \mid z_{-n}^{i-1}\right)\right)=0\end{cases}
\end{aligned}
$$

and $J_{\hat{v}}\left(z_{-n}^{i}\right)$ is similarly defined.

Note that

$$
x_{v, i+1}=\frac{x_{v, i} \Delta_{z_{i+1}}(\varepsilon)}{x_{v, i} \Delta_{z_{i+1}}(\varepsilon) \mathbf{1}} .
$$

Now with (4) and (5), we have

$$
\begin{aligned}
x_{v, i} \Delta_{z_{i+1}}(\varepsilon) & =\sum_{j=0}^{\infty} a_{v, j}\left(z_{-n}^{i}\right) \sum_{k=0}^{\infty} \frac{\Delta_{z_{i+1}}^{(k)}(0)}{k !} \varepsilon^{k} \\
& =\sum_{l=0}^{\infty} c_{v, l}\left(z_{-n}^{i+1}\right) \varepsilon^{l}
\end{aligned}
$$

where superscript ${ }^{(k)}$ denotes the $k$ th-order derivative with respect to $\varepsilon$.

We proceed by induction on $i$ (from $-n$ to -1 ).

First consider the case when $i=-n$. When $\max \left\{J_{v}\left(z_{-n}\right), J_{\hat{v}}\left(z_{-n}\right)\right\}>0$, the statement is vacuously true; when $J_{v}\left(z_{-n}\right)=J_{\hat{v}}\left(z_{-n}\right)=0$, necessarily $\Delta_{z_{-n}}(0)$ is a rank one matrix, $v(0) \Delta_{z_{-n}}(0) \mathbf{1}>0$ and $\hat{v}(0) \Delta_{z_{-n}}(0) 1>0$. Then we have

$a_{v, 0}\left(z_{-n}\right)=\frac{v(0) \Delta_{z_{-n}}(0)}{v(0) \Delta_{z_{-n}}(0) \mathbf{1}} \stackrel{(*)}{=} \frac{\hat{v}(0) \Delta_{z_{-n}}(0)}{\hat{v}(0) \Delta_{z_{-n}}(0) \mathbf{1}}=a_{\hat{v}, 0}\left(z_{-n}\right)$

where $(*)$ follows from the fact that $\Delta_{z_{-n}}(0)$ is a rank one matrix. (Similarly as in Remark 2.2, when $\Delta_{a}$ is a rank one matrix, the mapping $f_{a}(w)$ will map every $w \in W$ with $w \Delta_{a} \mathbf{1}>0$ to one single point.)

Now suppose $i \geq-n$ and that $a_{v, j}\left(z_{-n}^{i}\right)=a_{\hat{v}, j}\left(z_{-n}^{i}\right)$ for $j$ with $0 \leq j \leq n+i-\sum_{l=-n}^{i} \max \left\{J_{v}\left(z_{-n}^{l}\right), J_{\hat{v}}\left(z_{-n}^{l}\right)\right\}$.

If ord $\left(p_{v}\left(z_{i+1} \mid z_{-n}^{i}\right)\right)>0$, since the leading coefficient vector of the Taylor series expansion in (7) is nonnegative, $c_{v, j}\left(z_{-n}^{i+1}\right) \equiv \mathbf{0}$ for all $j$ with $0 \leq j \leq J_{v}\left(z_{-n}^{i+1}\right)-2$ and $c_{v, J_{v}\left(z_{-n}^{i+1}\right)-1}\left(z_{-n}^{i+1}\right) \not \equiv 0$. So applying Lemma 2.5 to the expression shown in (8) at the bottom of the next page, we conclude that for all $j, a_{v, j}\left(z_{-n}^{i+1}\right)$ depends only on

$$
c_{v, l}\left(z_{-n}^{i+1}\right), \quad J_{v}\left(z_{-n}^{i+1}\right)-1 \leq l \leq J_{v}\left(z_{-n}^{i+1}\right)-1+j,
$$

implying that $a_{v, j}\left(z_{-n}^{i+1}\right)$ depends only on (or some of)

$$
a_{v, l}\left(z_{-n}^{i}\right), \quad \Delta_{z_{i+1}}^{(l)}(0), \quad 0 \leq l \leq J_{v}\left(z_{-n}^{i+1}\right)-1+j .
$$

A completely parallel argument also applies to the case when ord $\left(p_{\hat{v}}\left(z_{i+1} \mid z_{-n}^{i}\right)\right)>0$; more specifically, the statements above for the case ord $\left(p\left(z_{i+1} \mid z_{-m}^{i}\right)\right)>0$ are still true if we replace $v$ with $\hat{v}$, which implies that $a_{\hat{v}, j}\left(z_{-n}^{i+1}\right)$ depends only on (or some of)

$$
a_{\hat{v}, l}\left(z_{-n}^{i}\right), \quad \Delta_{z_{i+1}}^{(l)}(0), \quad 0 \leq l \leq J_{\hat{v}}\left(z_{-n}^{i+1}\right)-1+j .
$$


Thus, when $\max \left\{J_{v}\left(z_{-n}^{i+1}\right), J_{\hat{v}}\left(z_{-n}^{i+1}\right)\right\}>0$, we have $a_{v, j}\left(z_{-n}^{i+1}\right)=a_{\hat{v}, j}\left(z_{-n}^{i+1}\right)$ for $j$ with

$$
\begin{aligned}
0 \leq & j \leq n+i-\sum_{l=-n}^{i} \max \left\{J_{v}\left(z_{-n}^{l}\right), J_{\hat{v}}\left(z_{-n}^{l}\right)\right\} \\
& -\max \left\{J_{v}\left(z_{-n}^{i+1}\right)-1, J_{\hat{v}}\left(z_{-n}^{i+1}\right)-1\right\} \\
= & n+(i+1)-\sum_{l=-n}^{i+1} \max \left\{J_{v}\left(z_{-n}^{l}\right), J_{\hat{v}}\left(z_{-n}^{l}\right)\right\} .
\end{aligned}
$$

If ord $\left(p_{v}\left(z_{i+1} \mid z_{-n}^{i}\right)\right)=0$, by (3) necessarily we have

$$
a_{v, 0}\left(z_{-n}^{i}\right) \Delta_{z_{i+1}}(0) 1 \neq 0 \text {. }
$$

Again by Lemma 2.5 applied to (8), for any $j, a_{v, j}\left(z_{-n}^{i+1}\right)$ depends only on

$$
a_{v, l}\left(z_{-n}^{i}\right), \quad \Delta_{z_{i+1}}^{(l)}(0), \quad 0 \leq l \leq j .
$$

Similarly, if ord $\left(p_{\hat{v}}\left(z_{i+1} \mid \hat{z}_{-n}^{i}\right)\right)=0$, we deduce that for any $j, a_{\hat{v}, j}\left(z_{-n}^{i+1}\right)$ depends only on

$$
a_{\hat{v}, l}\left(z_{-n}^{i}\right), \quad \Delta_{z_{i+1}}^{(l)}(0), \quad 0 \leq l \leq j .
$$

Thus, if $\max \left\{J_{v}\left(z_{-n}^{i+1}\right), J_{\hat{v}}\left(z_{-n}^{i+1}\right)\right\}=0$, for any $j$ with

$$
\begin{aligned}
0 & \leq j \leq n+i-\sum_{l=-n}^{i} \max \left\{J_{v}\left(z_{-n}^{l}\right), J_{\hat{v}}\left(z_{-n}^{l}\right)\right\} \\
& =n+i-\sum_{l=-n}^{i+1} \max \left\{J_{v}\left(z_{-n}^{l}\right), J_{\hat{v}}\left(z_{-n}^{l}\right)\right\}
\end{aligned}
$$

we have $a_{v, j}\left(z_{-n}^{i+1}\right)=a_{\hat{v}, j}\left(z_{-n}^{i+1}\right)$.

Now, let

$$
t=n+(i+1)-\sum_{l=-n}^{i+1} \max \left\{J_{v}\left(z_{-n}^{l}\right), J_{\hat{v}}\left(z_{-n}^{l}\right)\right\}
$$

Then one can show that [see the equation at the bottom of the page], where the first term in the expression above is equal to 0 (since $\Delta_{z_{i+1}}(0)$ is a rank one matrix), and the "other terms" are functions of

$$
a_{v, 0}\left(z_{-n}^{i}\right), \ldots, a_{v, t-1}\left(z_{-n}^{i}\right), \Delta_{z_{i+1}}^{(0)}(0), \ldots, \Delta_{z_{i+1}}^{(t)}(0) .
$$

It follows that $a_{v, j}\left(z_{-n}^{i+1}\right)$ is a function of the same quantities in (9). By a completely parallel argument as above, $a_{\hat{v}, j}\left(z_{-n}^{i+1}\right)$ is the same function of of the same quantities in (9). So we have $a_{v, j}\left(z_{-n}^{i+1}\right)=a_{\hat{v}, j}\left(z_{-n}^{i+1}\right)$ for $j$ with

$$
0 \leq j \leq n+(i+1)-\sum_{l=-n}^{i+1} \max \left\{J_{v}\left(z_{-n}^{l}\right), J_{\hat{v}}\left(z_{-n}^{l}\right)\right\}
$$

Notice that

$$
\begin{aligned}
\sum_{l=-n}^{-1} \max \left\{J_{v}\left(z_{-n}^{l}\right), J_{\hat{v}}\left(z_{-n}^{l}\right)\right\} & \\
& \leq \sum_{l=-n}^{-1}\left(J_{v}\left(z_{-n}^{l}\right)+J_{\hat{v}}\left(z_{-n}^{l}\right)\right) \leq 4 k .
\end{aligned}
$$

The lemma then immediately follows from (3) and the proven fact that $a_{v, j}\left(z_{-n}^{-1}\right)=a_{\hat{v}, j}\left(z_{-n}^{-1}\right)$ for $j$ with

$$
0 \leq j \leq n-1-\sum_{l=-n}^{-1} \max \left\{J_{v}\left(z_{-n}^{l}\right), J_{\hat{v}}\left(z_{-n}^{l}\right)\right\}
$$

By Proposition 2.4, for any hidden Markov string $z_{-m}^{0}$ (or $\left.z_{-\hat{m}}^{0}\right), p\left(y_{-n-1}=\cdot \mid z_{-m}^{-n-1}\right) \quad\left(\right.$ or $p\left(y_{-n-1}=\cdot \mid z_{-\hat{m}}^{-n-1}\right)$ ) is analytic. So for $n \leq m, \hat{m}$, if $v(\varepsilon)($ or $\hat{v}(\varepsilon))$ is equal to $p\left(y_{-n-1}=\cdot \mid z_{-m}^{-n-1}\right) \quad\left(\right.$ or $p\left(y_{-n-1}=\cdot \mid z_{-\hat{m}}^{-n-1}\right)$ ), then $p_{v}\left(z_{-n}^{0}\right)$ (or $p_{\hat{v}}\left(z_{-n}^{0}\right)$ ) will be equal to $p\left(z_{-n}^{0} \mid z_{-m}^{-n-1}\right)$ (or $\left.p\left(z_{-n}^{0} \mid z_{-\hat{m}}^{-n-1}\right)\right)$; and if for a Markov state $y, v(\varepsilon)$ (or $\hat{v}(\varepsilon)$ ) is equal to $p\left(y_{-n-1}=\cdot \mid z_{-m}^{-n-1} y\right)$ (or $p\left(y_{-n-1}=\cdot \mid z_{-\hat{m}}^{-n-1} y\right)$ ), then $p_{v}\left(z_{-n}^{0}\right)$ (or $\left.p_{\hat{v}}\left(z_{-n}^{0}\right)\right)$ will be equal to $p\left(z_{-n}^{0} \mid z_{-m}^{-n-1} y\right)$ (or $p\left(z_{-n}^{0} \mid z_{-\hat{m}}^{-n-1} y\right)$ ). In what follows, slightly abusing the notation, we use $b_{j}\left(z_{-m}^{0}\right)$ to represent the coefficient of $\varepsilon^{j}$ in the expansion of $p\left(z_{0} \mid z_{-m}^{-1}\right)$, namely

$$
p\left(z_{0} \mid z_{-m}^{-1}\right)=b_{0}\left(z_{-m}^{0}\right)+b_{1}\left(z_{-m}^{0}\right) \varepsilon+b_{2}\left(z_{-m}^{0}\right) \varepsilon^{2}+\cdots ;
$$

and we use $b_{j}\left(z_{-m}^{0} y_{-m-1}\right)$ to represent the coefficient of $\varepsilon^{j}$ in the expansion of $p\left(z_{0} \mid z_{-m}^{-1} y_{-m-1}\right)$, namely

$$
\begin{aligned}
& p\left(z_{0} \mid z_{-m}^{-1} y_{-m-1}\right)=b_{0}\left(z_{-m}^{0} y_{-m-1}\right) \\
& \quad+b_{1}\left(z_{-m}^{0} y_{-m-1}\right) \varepsilon+b_{2}\left(z_{-m}^{0} y_{-m-1}\right) \varepsilon^{2}+\cdots .
\end{aligned}
$$

$$
x_{v, i+1}=\frac{c_{v, 0}\left(z_{-n}^{i+1}\right)+c_{v, 1}\left(z_{-n}^{i+1}\right) \varepsilon+\cdots+c_{v, l}\left(z_{-n}^{i+1}\right) \varepsilon^{l}+\cdots}{c_{v, 0}\left(z_{-n}^{i+1}\right) \mathbf{1}+c_{v, 1}\left(z_{-n}^{i+1}\right) \mathbf{1} \varepsilon+\cdots+c_{v, l}\left(z_{-n}^{i+1}\right) \mathbf{1} \varepsilon^{l}+\cdots}=\frac{\sum_{l=0}^{\infty} c_{v, l+J_{v}\left(z_{-n}^{i+1}\right)-1}\left(z_{-n}^{i+1}\right) \varepsilon^{l}}{\sum_{l=0}^{\infty} c_{l+J_{v}\left(z_{-n}^{i+1}\right)-1}\left(z_{-n}^{i+1}\right) \mathbf{1} \varepsilon^{l}}
$$

$$
\begin{aligned}
a_{v, t}\left(z_{-n}^{i+1}\right)= & \frac{a_{v, t}\left(z_{-n}^{i}\right) \Delta_{z_{i+1}}(0) a_{v, 0}\left(z_{-n}^{i}\right) \Delta_{z_{i+1}}(0) \mathbf{1}-a_{v, 0}\left(z_{-n}^{i}\right) \Delta_{z_{i+1}}(0) a_{v, t}\left(z_{-n}^{i}\right) \Delta_{z_{i+1}}(0) \mathbf{1}}{\left(a_{v, 0}\left(z_{-n}^{i}\right) \Delta_{z_{i+1}}(0) \mathbf{1}\right)^{2}} \\
& + \text { other terms }
\end{aligned}
$$


It then immediately follows from Lemma 2.6 that [see Proposition 2.7].

Proposition 2.7: Given fixed sequences $z_{-m}^{0}, z_{-\hat{m}}^{0}$, $z_{-m}^{0} y_{-m-1}, \hat{z}_{-\hat{m}}^{0} y_{-\hat{m}-1}$ with $z_{-n}^{0}=\hat{z}_{-n}^{0}$ such that

ord $\left(p\left(z_{-n}^{-1} \mid z_{-m}^{-n-1}\right)\right)$, ord $\left(p\left(\hat{z}_{-n}^{-1} \mid \hat{z}_{-\hat{m}}^{-n-1}\right)\right)$

ord $\left(p\left(z_{-n}^{-1} \mid z_{-m}^{-n-1} y_{-m-1}\right)\right)$, ord $\left(p\left(\hat{z}_{-n}^{-1} \mid \hat{z}_{-\hat{m}}^{-n-1} y_{-\hat{m}-1}\right)\right) \leq k$

for $n \leq m, \hat{m}$ and some $k$, we have for $j$ with $0 \leq j \leq n-4 k-1$

$b_{j}\left(z_{-m}^{0} y_{-m-1}\right)=b_{j}\left(\hat{z}_{-\hat{m}}^{0} y_{-\hat{m}-1}\right)=b_{j}\left(z_{-m}^{0}\right)=b_{j}\left(\hat{z}_{-\hat{m}}^{0}\right)$.

Consider (10). In the following, we use $p^{\langle l\rangle}\left(z_{0} \mid z_{-n}^{-1}\right)$ to denote the truncated (up to the $(l+1)$-st term) Taylor series expansion of $p\left(z_{0} \mid z_{-n}^{-1}\right)$, i.e.

$$
p^{\langle l\rangle}\left(z_{0} \mid z_{-n}^{-1}\right)=b_{0}\left(z_{-n}^{0}\right)+b_{1}\left(z_{-n}^{0}\right) \varepsilon+\cdots+b_{l}\left(z_{-n}^{0}\right) \varepsilon^{l} .
$$

Theorem 2.8: For a hidden Markov chain $Z$ with normally parameterized $\Delta(\varepsilon)$, we have for any $k \geq 0$,

$$
H(Z)=\left.H(Z)\right|_{\varepsilon=0}+\sum_{j=1}^{k+1} f_{j} \varepsilon^{j} \log \varepsilon+\sum_{j=1}^{k} g_{j} \varepsilon^{j}+O\left(\varepsilon^{k+1}\right)
$$

where $f_{j}$ 's and $g_{j}$ 's for $j=1,2, \ldots, k+1$ are functions (more specifically, elementary functions built from log and polynomials) of $\Delta^{(i)}(0)$ for $0 \leq i \leq 6 k+6$ and can be computed from $H_{6 k+6}(Z(\varepsilon))$.

The following theorem [3] states the Birch upper bound and lower bound of $H(Z)$, which we shall use in the proof of Theorem 2.8 .

Theorem 2.9 (Birch, 1962): For any $n$,

$$
H\left(Z_{0} \mid Z_{-n}^{-1} Y_{-n-1}\right) \leq H(Z) \leq H\left(Z_{0} \mid Z_{-n}^{-1}\right) .
$$

Proof of Theorem 2.8: First fix $n$ such that $n \geq n_{0}=$ $6 k+6$. Consider the Birch upper bound on $H(Z)$

$$
H_{n}(Z):=H\left(Z_{0} \mid Z_{-n}^{-1}\right)=-\sum_{z_{-n}^{0}} p\left(z_{-n}^{0}\right) \log p\left(z_{0} \mid z_{-n}^{-1}\right) .
$$

Note that for $j \geq k+2$,

$$
\left|\sum_{\operatorname{ord}\left(p\left(z_{-n}^{0}\right)\right)=j} p\left(z_{-n}^{0}\right) \log p\left(z_{0} \mid z_{-n}^{-1}\right)\right|=O\left(\varepsilon^{k+1}\right) .
$$

(We used simplified notation above: $\sum_{z_{-n}^{0}}$ means summation over all $z_{-n}^{0} \in \mathcal{A}^{n+1}$, while $\sum_{\text {ord }\left(p\left(z_{-n}^{0}\right)\right)=j}$ means summation over all $z_{-n}^{0} \in \mathcal{A}^{n+1}$ with ord $\left(p\left(z_{-n}^{0}\right)\right)=j$; the same notational convention will be followed in the rest of the proof.) So, in the following we only consider the sequences $z_{-n}^{0}$ with ord $\left(p\left(z_{-n}^{0}\right)\right) \leq k+1$. For such sequences, since ord $\left(p\left(z_{0} \mid z_{-n}^{-1}\right)\right) \leq$ ord $\left(p\left(z_{-n}^{0}\right)\right) \leq k+1$, we have

$$
\left|\log p\left(z_{0} \mid z_{-n}^{-1}\right)-\log p^{\langle 2 k+1\rangle}\left(z_{0} \mid z_{-n}^{-1}\right)\right|=O\left(\varepsilon^{k+1}\right) ;
$$

and by Lemma 2.6, we have

$$
p^{\langle 2 k+1\rangle}\left(z_{0} \mid z_{-n}^{-1}\right)=p^{\langle 2 k+1\rangle}\left(z_{0} \mid z_{-n_{0}}^{-1}\right) .
$$

Now for any fixed $n \geq n_{0}$

$$
\begin{aligned}
& H_{n}(Z)=\sum_{z_{-n}^{0}}-p\left(z_{-n}^{0}\right) \log p\left(z_{0} \mid z_{-n}^{-1}\right) \\
& \stackrel{(a)}{=} \sum_{\operatorname{ord}\left(p\left(z_{-n}^{0}\right)\right) \leq k+1}-p\left(z_{-n}^{0}\right) \log p\left(z_{0} \mid z_{-n}^{-1}\right) \\
& +O\left(\varepsilon^{k+1}\right) \\
& \stackrel{(b)}{=} \sum_{\operatorname{ord}\left(p\left(z_{-n}^{0}\right)\right) \leq k+1}-p\left(z_{-n}^{0}\right) \log p^{\langle 2 k+1\rangle}\left(z_{0} \mid z_{-n}^{-1}\right) \\
& +O\left(\varepsilon^{k+1}\right) \\
& \stackrel{(c)}{=} \sum_{\text {ord }\left(p\left(z_{-n_{0}}^{0}\right)\right) \leq k+1}-p\left(z_{-n}^{0}\right) \log p^{\langle 2 k+1\rangle}\left(z_{0} \mid z_{-n_{0}}^{-1}\right) \\
& +O\left(\varepsilon^{k+1}\right) \\
& =\sum_{\text {ord }\left(p\left(z_{-n_{0}}^{0}\right)\right) \leq k+1}-p\left(z_{-n_{0}}^{0}\right) \log p^{\langle 2 k+1\rangle}\left(z_{0} \mid z_{-n_{0}}^{-1}\right) \\
& +O\left(\varepsilon^{k+1}\right)
\end{aligned}
$$

where (a) follows from (14); (b) follows from (15); (c) follows from (16), (14) and the fact that

$\left\{z_{-n}^{0}:\right.$ ord $\left.\left(p\left(z_{-n_{0}}^{0}\right)\right) \leq k+1\right\}$

$=\left\{z_{-n}^{0}:\right.$ ord $\left.\left(p\left(z_{-n}^{0}\right)\right) \leq k+1\right\}$

$\cup\left\{z_{-n}^{0}\right.$ : ord $\left(p\left(z_{-n_{0}}^{0}\right)\right) \leq k+1$, ord $\left.\left(p\left(z_{-n}^{0}\right)\right) \geq k+2\right\}$.

Expanding (17), we obtain

$$
H_{n}(Z)=\left.H(Z)\right|_{\varepsilon=0}+\sum_{j=1}^{k+1} f_{j} \varepsilon^{j} \log \varepsilon+\sum_{j=1}^{k} g_{j} \varepsilon^{j}+O\left(\varepsilon^{k+1}\right)
$$

where $f_{j}$ 's and $g_{j}$ 's for $j=1,2, \ldots, k+1$ are functions dependent only on $\Delta^{(i)}(0)$ for $0 \leq i \leq n_{0}$ and can be computed from $H_{n_{0}}(Z)$ (in fact for fixed $j, f_{j}$ and $g_{j}$ are functions dependent only on $\Delta^{(i)}(0)$ for $0 \leq i \leq 6 j+6$ and can be computed from $H_{6 j+6}(Z)$ ). In particular, [see (18) at the bottom of the next page] will contribute to $\left.H(Z)\right|_{\varepsilon=0}$ and the terms $\varepsilon^{j}$, and [see (19) at the bottom of the next page] will contribute to the terms $\varepsilon^{j} \log \varepsilon$ and the terms $\varepsilon^{j}$.

Using Corollary 2.7, one can apply similar argument as above to the Birch lower bound

$$
\begin{aligned}
\tilde{H}_{n}(Z) & :=H\left(Z_{0} \mid Z_{-n}^{-1} Y_{-n-1}\right) \\
& =\sum_{z_{-n}^{0}, y_{-n-1}}-p\left(z_{-n}^{0} y_{-n-1}\right) \log p\left(z_{0} \mid z_{-n}^{-1} y_{-n-1}\right) .
\end{aligned}
$$

For the same $n_{0}$, one can show that $\tilde{H}_{n}(Z)$ takes the same form (17) as $H_{n}(Z)$, which implies that $H_{n}(Z)$ and $\tilde{H}_{n}(Z)$ have exactly the same coefficients of $\varepsilon^{j}$ for $j \leq k$ and of $\varepsilon^{j} \log \varepsilon$ for $j \leq k+1$ when $n \geq n_{0}$. We thus prove the theorem. 
Remark 2.10: Theorem 2.8 still holds if we assume each entry of $\Delta(\varepsilon)$ is merely a $C^{6 k+6}$ function of $\varepsilon$ in a neighborhood of $\varepsilon=0$ : the proof still works if "analytic" is replaced by " $C^{6 k+6}$," and the Taylor series expansions are replaced by Taylor polynomials with remainder. We assumed analyticity of the parametrization only for simplicity.

Remark 2.11: Note that at a Black Hole, we have ord $\left(p\left(z_{0} \mid z_{-n}^{-1}\right)\right)=0$ for any hidden Markov symbol sequence $z_{-n}^{0}$. Thus, from the discussion surrounding (18) and (19) above, we see that $f_{j}=0$ for all $j$. By the proof of Theorem 2.8, (13) is a Taylor polynomial with remainder; this is consistent with the Taylor series formula for a Black Hole in [8].

\section{Applications to Finite-State Memoryless Channels AT High Signal-TO-Noise RATio}

Consider a finite-state memoryless channel with stationary input process. Here, $C=\left\{C_{n}\right\}$ is an i.i.d. channel state process over finite alphabet $\mathcal{C}$ with $p_{C}(c)=q_{c}$ for $c \in \mathcal{C}, X=\left\{X_{n}\right\}$ is a stationary input process, independent of $C$, over finite alphabet $\mathcal{X}$ and $Z=\left\{Z_{n}\right\}$ is the resulting (stationary) output process over finite alphabet $\mathcal{Z}$. Let $p\left(z_{n} \mid x_{n}, c_{n}\right)=P\left(Z_{n}=z_{n} \mid X_{n}=\right.$ $x_{n}, C_{n}=c_{n}$ ) denote the probability that at time $n$, the channel output symbol is $z_{n}$ given that the channel state is $c_{n}$ and the channel input is $x_{n}$. The mutual information for such a channel is

$$
\begin{aligned}
I(X, Z) & :=H(Z)-H(Z \mid X) \\
& \stackrel{(*)}{=} H(Z)-\sum_{x \in \mathcal{X}, z \in \mathcal{Z}} p(x, z) \log p(z \mid x)
\end{aligned}
$$

where $(*)$ follows from the memoryless property of the channel, and for $x \in \mathcal{X}, z \in \mathcal{Z}$

$$
\begin{aligned}
& p(x, z)=\sum_{c \in \mathcal{C}} p(z \mid x, c) p(x) p(c) \\
& p(z \mid x)=\sum_{c \in \mathcal{C}} p(z \mid x, c) p(c) .
\end{aligned}
$$

Now we introduce an alternative framework, using the concept of channel noise. As above, let $C$ be an i.i.d. channel state process, and let $X$ be a stationary input process, independent of
$C$, over finite alphabets $\mathcal{C}, \mathcal{X}$. Let $\mathcal{E}$ (respectively, $\mathcal{Z}$ ) be finite alphabets of abstract error events (respectively, output symbols) and let $\Phi: \mathcal{X} \times \mathcal{C} \times \mathcal{E} \rightarrow \mathcal{Z}$ be a function. For each $x \in \mathcal{X}$ and $c \in \mathcal{C}$, let $p(\cdot \mid x, c)$ be a conditional probability distribution on $\mathcal{E}$. This defines a jointly distributed stationary process $(X, C, E)$ over $\mathcal{X} \times \mathcal{C} \times \mathcal{E}$. If $X$ is a first-order Markov chain with transition probability matrix $\Pi$, then $(X, C, E)$ is a Markov chain with transition probability matrix $\Delta$, defined by

$$
\Delta_{(x, c, e),(y, d, f)}=\Pi_{x y} \cdot q_{d} \cdot p(f \mid y, d)
$$

and $\Phi, \Delta$ define a hidden Markov chain, denoted $Z(\Delta, \Phi)$.

We claim that the output process $Z$, described in the first paragraph of this section, fits into this alternative framework (when $X$ is a first-order Markov chain). To see this, let $\mathcal{E}=\mathcal{X} \times \mathcal{C} \times \mathcal{Z}$, and define $p\left(e=(x, c, z) \mid x^{\prime}, c^{\prime}\right)=p(z \mid x, c)$ if $x=x^{\prime}$ and $c=c^{\prime}$, and 0 otherwise. Define $\Phi\left(x^{\prime}, c^{\prime},(x, c, z)\right)=z$. Then, $Z=Z(\Delta, \Phi)$ is a hidden Markov chain. So, from hereon we adopt the alternative framework.

Now, we assume that $X$ is an irreducible first-order Markov chain and that the channel is parameterized by $\varepsilon$ such that for each $x, c$, and $e, p(e \mid x, c)(\varepsilon)$ are analytic functions of $\varepsilon \geq 0$. For each $\varepsilon \geq 0$, let $\Delta(\varepsilon)$ denote the corresponding transition probability matrix on state set $\mathcal{X} \times \mathcal{C} \times \mathcal{E}$ and $\{Z(\varepsilon)\}$ denote the family of resulting output hidden Markov chains. We also assume that there is a one-to-one function from $\mathcal{X}$ into $\mathcal{Z}, z=z(x)$, such that for all $c, p(z(x) \mid x, c)(0)=1$. In other words, $\varepsilon$ behaves like a "composite index" indicating how good the channel is, and small $\varepsilon$ corresponds to the high signal-to-noise ratio. Then one can verify that $\Delta(0)$ is a weak black hole and $\Delta(\varepsilon)$ is normally parameterized. Thus, by Theorem 2.8 , we obtain an asymptotic formula for $H(Z(\varepsilon))$ around $\varepsilon=0$. We remark that the above naturally generalizes to the case where $X$ is a higher-order irreducible Markov chain (through appropriately grouping matrices into blocks).

In the remainder of this section, we give three examples to illustrate the idea.

Example 3.1: [Binary Markov Chains Corrupted by BSC $(\varepsilon)$ ]

Consider a binary symmetric channel with crossover probability $\varepsilon$. At time $n$ the channel can be characterized by the following:

$$
Z_{n}=X_{n} \oplus E_{n}
$$

$$
\sum_{\operatorname{ord}\left(p\left(z_{-n}^{0}\right)\right) \leq k+1 \text { ord }\left(p\left(z_{0} \mid z_{-n_{0}}^{-1}\right)\right)=0}-p\left(z_{-n_{0}}^{0}\right) \log p^{\langle 2 k+1\rangle}\left(z_{0} \mid z_{-n_{0}}^{-1}\right)
$$

$$
\sum_{\operatorname{ord}\left(p\left(z_{-n}^{0}\right)\right) \leq k+1 \text { ord }\left(p\left(z_{0} \mid z_{-n_{0}}^{-1}\right)\right)>0}-p\left(z_{-n_{0}}^{0}\right) \log p^{\langle 2 k+1\rangle}\left(z_{0} \mid z_{-n_{0}}^{-1}\right)
$$


where $\left\{X_{n}\right\}$ denotes the input process, $\oplus$ denotes binary addition, $\left\{E_{n}\right\}$ denotes the i.i.d. binary noise with $p_{E}(0)=1-\varepsilon$ and $p_{E}(1)=\varepsilon$, and $\left\{Z_{n}\right\}$ denotes the corrupted output. Note that this channel only has one channel state, and at $\varepsilon=0$, $p_{Z \mid X}(1 \mid 1)=1, p_{Z \mid X}(0 \mid 0)=1$, so it fits in the alternative framework described in the beginning of Section III.

Indeed, suppose $X$ is a first-order irreducible Markov chain with the transition probability matrix

$$
\Pi=\left[\begin{array}{ll}
\pi_{00} & \pi_{01} \\
\pi_{10} & \pi_{11}
\end{array}\right] .
$$

Then $Y=\left\{Y_{n}\right\}=\left\{\left(X_{n}, E_{n}\right)\right\}$ is jointly Markov with transition probability matrix (the column and row indices of the following matrix are ordered alphabetically)

$$
\Delta=\left[\begin{array}{cccc}
\pi_{00}(1-\varepsilon) & \pi_{00} \varepsilon & \pi_{01}(1-\varepsilon) & \pi_{01} \varepsilon \\
\pi_{00}(1-\varepsilon) & \pi_{00} \varepsilon & \pi_{01}(1-\varepsilon) & \pi_{01} \varepsilon \\
\pi_{10}(1-\varepsilon) & \pi_{10} \varepsilon & \pi_{11}(1-\varepsilon) & \pi_{11} \varepsilon \\
\pi_{10}(1-\varepsilon) & \pi_{10} \varepsilon & \pi_{11}(1-\varepsilon) & \pi_{11} \varepsilon
\end{array}\right]
$$

and $Z=\Phi(Y)$ is a hidden Markov chain with $\Phi(0,0)=$ $\Phi(1,1)=0, \Phi(0,1)=\Phi(1,0)=1$. When $\varepsilon=0$,

$$
\begin{aligned}
\Delta & =\left[\begin{array}{llll}
\pi_{00} & 0 & \pi_{01} & 0 \\
\pi_{00} & 0 & \pi_{01} & 0 \\
\pi_{10} & 0 & \pi_{11} & 0 \\
\pi_{10} & 0 & \pi_{11} & 0
\end{array}\right], \Delta_{0}=\left[\begin{array}{llll}
\pi_{00} & 0 & 0 & 0 \\
\pi_{00} & 0 & 0 & 0 \\
\pi_{10} & 0 & 0 & 0 \\
\pi_{10} & 0 & 0 & 0
\end{array}\right] \\
\Delta_{1} & =\left[\begin{array}{llll}
0 & 0 & \pi_{01} & 0 \\
0 & 0 & \pi_{01} & 0 \\
0 & 0 & \pi_{11} & 0 \\
0 & 0 & \pi_{11} & 0
\end{array}\right]
\end{aligned}
$$

thus both $\Delta_{0}$ and $\Delta_{1}$ have rank one. If $\pi_{i j}$ 's are all positive, then we have a Black Hole case, for which one can derive the Taylor series expansion of $H(Z)$ around $\varepsilon=0$ [8], [21]; if $\pi_{00}$ or $\pi_{11}$ are zero, then this is a weak Black hole case with normal parameterization (of $\varepsilon$ ), for which Theorem 2.8 can be applied and an asymptotic formula for $H(Z)$ around $\varepsilon=0$ can be derived.

For a first-order Markov chain $X$ with the following transition probability matrix

$$
\left[\begin{array}{cc}
1-p & p \\
1 & 0
\end{array}\right]
$$

where $0 \leq p \leq 1$, it has been shown [17] that

$$
H(Z)=H(X)-\frac{p(2-p)}{1+p} \varepsilon \log \varepsilon+O(\varepsilon)
$$

as $\varepsilon \rightarrow 0$. This result has been further generalized [9], [10], [14] to the following:

$$
H(Z)=H(X)+f(X) \varepsilon \log (1 / \varepsilon)+g(X) \varepsilon+O\left(\varepsilon^{2} \log \varepsilon\right)
$$

where $X$ is the input Markov chain of any order $m$ with transition probabilities $P\left(X_{t}=a_{0} \mid X_{t-m}^{t-1}=a_{-m}^{-1}\right), a_{-m}^{0} \in \mathcal{X}^{m}$, where $\mathcal{X}=\{0,1\}, Z$ is the output process obtained by passing $X$ through a BSC $(\varepsilon)$, and $f(X)$ and $g(X)$ can be explicitly computed. Theorem 2.8 can be used to generalize (20) to a formula with higher asymptotic terms. In particular, when $P\left(X_{t}=a_{0} \mid X_{t-m}^{t-1}=a_{-m}^{-1}\right)>0$ for $a_{-m}^{0} \in \mathcal{X}^{m+1}$, we define an augmented Markov chain $\left\{\tilde{X}_{i}, i \in \mathbb{Z}\right\}$ by

$$
\tilde{X}_{i}=\left(X_{m i}, X_{m i+1}, \ldots, X_{m i+m-1}\right) ;
$$

correspondingly we have output process $\tilde{Z}=\left\{\tilde{Z}_{i}, i \in \mathbb{Z}\right\}$, where

$$
\tilde{Z}_{i}=\left(Z_{m i}, Z_{m i+1}, \ldots, Z_{m i+m-1}\right) .
$$

Then one check that for this augmented hidden Markov chain, we have a Black Hole at $\varepsilon=0$, which implies that the Taylor series expansions of $H(Z)=H(\tilde{Z}) / m$ around $\varepsilon=0$ can be explicitly computed (in principle); similarly when $P\left(X_{t}=a_{0} \mid X_{t-m}^{t-1}=a_{-m}^{-1}\right)=0$ for some $a_{-m}^{0} \in \mathcal{X}^{m+1}$, we have a weak Black Hole, in which case an asymptotic formula of $H(Z)$ around $\varepsilon=0$ can be obtained.

Example 3.2: [Binary Markov Chains Corrupted by BEC $(\varepsilon)$ ]

Consider a BEC $(\varepsilon)$, i.e., a binary erasure channel with fixed erasure rate $\varepsilon$. At time $n$ the channel can be characterized by

$$
Z_{n}= \begin{cases}X_{n} & \text { if } E_{n}=0 \\ e & \text { if } E_{n}=1\end{cases}
$$

where $\left\{X_{n}\right\}$ denotes the input process, $e$ denotes the erasure, $\left\{E_{n}\right\}$ denotes the i.i.d. binary noise with $p_{E}(0)=1-\varepsilon$ and $p_{E}(1)=\varepsilon$, and $\left\{Z_{n}\right\}$ denotes the corrupted output. Again this channel only has one channel state, and at $\varepsilon=0, p_{Z \mid X}(1 \mid 1)=$ $1, p_{Z \mid X}(0 \mid 0)=1$, so it fits in the alternative framework described in the beginning of Section III.

If the input $X$ is a first-order irreducible Markov chain with transition probability matrix

$$
\Pi=\left[\begin{array}{ll}
\pi_{00} & \pi_{01} \\
\pi_{10} & \pi_{11}
\end{array}\right]
$$

and let $Z$ denote the output process. Then $Y=(X, E)$ is jointly Markov with (the column and row indices of the following matrix are ordered alphabetically)

$$
\Delta=\left[\begin{array}{llll}
\pi_{00}(1-\varepsilon) & \pi_{00} \varepsilon & \pi_{01}(1-\varepsilon) & \pi_{01} \varepsilon \\
\pi_{00}(1-\varepsilon) & \pi_{00} \varepsilon & \pi_{01}(1-\varepsilon) & \pi_{01} \varepsilon \\
\pi_{10}(1-\varepsilon) & \pi_{10} \varepsilon & \pi_{11}(1-\varepsilon) & \pi_{11} \varepsilon \\
\pi_{10}(1-\varepsilon) & \pi_{10} \varepsilon & \pi_{11}(1-\varepsilon) & \pi_{11} \varepsilon
\end{array}\right]
$$

and $Z=\Phi(Y)$ is hidden Markov with $\Phi(0,1)=\Phi(1,1)=e$, $\Phi(0,0)=0$ and $\Phi(1,0)=1$.

Now one checks that

$$
\begin{aligned}
& \Delta_{0}=\left[\begin{array}{llll}
\pi_{00}(1-\varepsilon) & 0 & 0 & 0 \\
\pi_{00}(1-\varepsilon) & 0 & 0 & 0 \\
\pi_{10}(1-\varepsilon) & 0 & 0 & 0 \\
\pi_{10}(1-\varepsilon) & 0 & 0 & 0
\end{array}\right] \\
& \Delta_{1}=\left[\begin{array}{llll}
0 & 0 & \pi_{01}(1-\varepsilon) & 0 \\
0 & 0 & \pi_{01}(1-\varepsilon) & 0 \\
0 & 0 & \pi_{11}(1-\varepsilon) & 0 \\
0 & 0 & \pi_{11}(1-\varepsilon) & 0
\end{array}\right] \\
& \Delta_{e}=\left[\begin{array}{llll}
0 & \pi_{00} \varepsilon & 0 & \pi_{01} \varepsilon \\
0 & \pi_{00} \varepsilon & 0 & \pi_{01} \varepsilon \\
0 & \pi_{10} \varepsilon & 0 & \pi_{11} \varepsilon \\
0 & \pi_{10} \varepsilon & 0 & \pi_{11} \varepsilon
\end{array}\right] .
\end{aligned}
$$




$$
\Delta=\left[\begin{array}{llllllll}
\pi_{00} q_{0}\left(1-\varepsilon_{0}\right) & \pi_{00} q_{0} \varepsilon_{0} & \pi_{00} q_{1}\left(1-\varepsilon_{1}\right) & \pi_{00} q_{1} \varepsilon_{1} & \pi_{01} q_{0}\left(1-\varepsilon_{0}\right) & \pi_{01} q_{0} \varepsilon_{0} & \pi_{01} q_{1}\left(1-\varepsilon_{1}\right) & \pi_{01} q_{1} \varepsilon_{1} \\
\pi_{10} q_{0}\left(1-\varepsilon_{0}\right) & \pi_{10} q_{0} \varepsilon_{0} & \pi_{10} q_{1}\left(1-\varepsilon_{1}\right) & \pi_{10} q_{1} \varepsilon_{1} & \pi_{11} q_{0}\left(1-\varepsilon_{0}\right) & \pi_{11} q_{0} \varepsilon_{0} & \pi_{11} q_{1}\left(1-\varepsilon_{1}\right) & \pi_{11} q_{1} \varepsilon_{1}
\end{array}\right] \otimes \mathbf{1}_{4}
$$

One checks that $\Delta(\varepsilon)$ is normally parameterized by $\varepsilon$ and thus Theorem 2.8 can be applied. Furthermore, Theorem 2.8 can be applied to the case when the input is an $m$ th-order irreducible Markov chain $X$ to obtain asymptotic formula for $H(Z)$ around $\varepsilon=0$.

Example 3.3: (Binary Markov Chains Corrupted by Special Gilbert-Elliot Channel)

Consider a binary Gilbert-Elliot channel, whose channel state (denoted by $C=\left\{C_{n}\right\}$ ) varies as an i.i.d. binary stochastic process with $p_{C}(0)=q_{0}, p_{C}(1)=q_{1}$ (here the channel state varies as an i.i.d. process, rather than a generic Markov process). At time $n$ the channel can be characterized by the

$$
Z_{n}=X_{n} \oplus E_{n}
$$

where $\left\{X_{n}\right\}$ denotes the input process, $\oplus$ denotes binary addition, $\left\{E_{n}\right\}$ denotes the i.i.d. binary noise with $p_{E \mid C}(0 \mid 0)=$ $1-\varepsilon_{0}, p_{E \mid C}(0 \mid 1)=1-\varepsilon_{1}, p_{E \mid C}(1 \mid 0)=\varepsilon_{0}, p_{E \mid C}(1 \mid 1)=\varepsilon_{1}$ and $\left\{Z_{n}\right\}$ denotes the corrupted output. For such a channel, $p_{Z \mid(X, C)}(1 \mid 1, c)=1, p_{Z \mid(X, C)}(0 \mid 0, c)=1$ at $\varepsilon=0$ for any channel state $c$. So it fits in the alternative framework described in the beginning of Section III.

To see this in more detail, we consider the special case when the input $X$ is a first-order irreducible Markov chain with transition probability matrix

$$
\Pi=\left[\begin{array}{ll}
\pi_{00} & \pi_{01} \\
\pi_{10} & \pi_{11}
\end{array}\right]
$$

and let $Z$ denote the output process. Then $Y=(X, C, E)$ is jointly Markov with transition probability matrix $\Delta$, which can be concisely written using Kronecker product as follows (the column and row indices of the following matrix are ordered alphabetically) (see the equation at the top of the page) where $1_{4}$ stands for the all one column vector of length 4 . $Z=\Phi(X, C, E)$ is hidden Markov with

$$
\begin{aligned}
& \Phi(0,0,0)=\Phi(0,1,0)=\Phi(1,0,1)=\Phi(1,1,1)=0 \\
& \Phi(0,0,1)=\Phi(0,1,1)=\Phi(1,0,0)=\Phi(1,1,0)=1
\end{aligned}
$$

For some positive $k$, let $\varepsilon_{0}=\varepsilon, \varepsilon_{1}=k \varepsilon$. If $\varepsilon=0$, one checks that

$$
\begin{aligned}
\Delta_{0} & =\left[\begin{array}{llllllll}
\pi_{00} q_{0} & 0 & \pi_{00} q_{1} & 0 & 0 & 0 & 0 & 0 \\
\pi_{10} q_{0} & 0 & \pi_{10} q_{1} & 0 & 0 & 0 & 0 & 0
\end{array}\right] \otimes \mathbf{1}_{4} \\
\Delta_{1} & =\left[\begin{array}{llllllll}
0 & 0 & 0 & 0 & \pi_{01} q_{0} & 0 & \pi_{01} q_{1} & 0 \\
0 & 0 & 0 & 0 & \pi_{11} q_{0} & 0 & \pi_{11} q_{1} & 0
\end{array}\right] \otimes \mathbf{1}_{4} .
\end{aligned}
$$

So, both $\Delta_{0}$ and $\Delta_{1}$ will be rank one matrices and one can check that $\Delta(\varepsilon)$ is normally parameterized by $\varepsilon$. Again, Theorem 2.8 can be applied to the case when the input is an $m$ th-order irreducible Markov chain $X$ to obtain an asymptotic formula for $H(Z)$ around $\varepsilon=0$.

\section{REFERENCES}

[1] D. Arnold and H. Loeliger, "The information rate of binary-input channels with memory," in Proc. 2001 IEEE Int. Conf. Commun., Helsinki, Finland, Jun. 11-14, 2001, pp. 2692-2695.

[2] D. M. Arnold, H.-A. Loeliger, P. O. Vontobel, A. Kavcic, and W. Zeng, "Simulation-based computation of information rates for channels with memory," IEEE Trans. Inf. Theory, vol. 52, pp. 3498-3508, 2006.

[3] J. Birch, "Approximations for the entropy for functions of Markov chains," Ann. Math. Statist., vol. 33, pp. 930-938, 1962.

[4] D. Blackwell, "The entropy of functions of finite-state Markov chains," in Trans. First Prague Conf. Inf. Thoery, Statistical Decision Functions, Random Processes, 1957, pp. 13-20.

[5] S. Egner, V. Balakirsky, L. Tolhuizen, S. Baggen, and H. Hollmann, "On the entropy rate of a hidden Markov model," in Proc. 2004 IEEE Int. Symp. Inf. Theory, Chicago, IL, Jun. 2, 2004, pp. 12-12.

[6] R. Gharavi and V. Anantharam, "An upper bound for the largest Lyapunov exponent of a Markovian product of nonnegative matrices," Theoret. Comput. Sci., vol. 332, no. 1-3, pp. 543-557, Feb. 2005.

[7] G. Han and B. Marcus, "Analyticity of entropy rate of hidden Markov chains," IEEE Trans. Inf. Theory, vol. 52, pp. 5251-5266, Dec. 2006.

[8] G. Han and B. Marcus, "Derivatives of entropy rate in special families of hidden Markov chains," IEEE Trans. Inf. Theory, vol. 53, pp. 2642-2652, Jul. 2007.

[9] G. Han and B. Marcus, "Asymptotics of noisy constrained capacity," in Proc. ISIT 2007, Nice, Jun. 29, 2007, pp. 991-995.

[10] G. Han and B. Marcus, "Asymptotics of input-constrained binary symmetric channel capacity," Ann. Appl. Probabil., vol. 19, no. 3, pp. 1063-1091, Jun. 2009.

[11] T. Holliday, A. Goldsmith, and P. Glynn, "Capacity of finite state Markov channels with general inputs," in Proc. 2003 IEEE Int. Symp. Inf. Theory, Jul. 29, 2003, pp. 289-289.

[12] T. Holliday, A. Goldsmith, and P. Glynn, "Capacity of finite state channels based on Lyapunov exponents of random matrices," IEEE Trans. Inf. Theory, vol. 52, pp. 3509-3532, Aug. 2006.

[13] P. Jacquet, G. Seroussi, and W. Szpankowski, "On the entropy of a hidden Markov process (extended abstract)," in Data Compression Conf., Snowbird, UT, 2004, pp. 362-371.

[14] P. Jacquet, G. Seroussi, and W. Szpankowski, "Noisy constrained capacity," in Proc. Int. Symp. Inf. Theory, Nice, 2007, pp. 986-990.

[15] D. Lind and B. Marcus, An Introduction to Symbolic Dynamics and Coding. Cambridge, U.K.: Cambridge Univ. Press, 1995.

[16] E. Ordentlich and T. Weissman, "On the optimality of symbol by symbol filtering and denoising," IEEE Trans. Inf. Theory, vol. 52, no. 1, pp. 19-40, Jan. 2006.

[17] E. Ordentlich and T. Weissman, "New bounds on the entropy rate of hidden Markov process," in Proc. IEEE Inf. Theory Workshop, San Antonio, TX, Oct. 24-29, 2004, pp. 117-122.

[18] H. Pfister, J. Soriaga, and P. Siegel, "The achievable information rates of finite-state ISI channels," in Proc. IEEE GLOBECOM, San Antonio, TX, Nov. 2001, pp. 2992-2996.

[19] E. Seneta, Non-Negative Matrices and Markov Chains, ser. Springer Series in Statistics. New York: Springer-Verlag, 1980.

[20] V. Sharma and S. Singh, "Entropy and channel capacity in the regenerative setup with applications to Markov channels," in Proc. IEEE Int. Symp. Inf. Theory, Washington, DC, Jun. 24-29, 2001, pp. 283-283.

[21] O. Zuk, I. Kanter, and E. Domany, "The entropy of a binary hidden Markov process," J. Stat. Phys., vol. 121, no. 3-4, pp. 343-360, 2005.

[22] O. Zuk, E. Domany, I. Kanter, and M. Aizenman, "From finite-system entropy to entropy rate for a hidden Markov process," IEEE Signal Process. Lett., vol. 13, no. 9, pp. 517-520, Sep. 2006. 
Guangyue Han (M'08) received the B.S. and M.S. degrees in mathematics from Peking University, China, and the Ph.D. degree in mathematics from University of Notre Dame, Notre Dame, IN, in 1997, 2000, and 2004, respectively.

After three years with the Department of Mathematics, University of British Columbia, Canada, he joined the Department of Mathematics, University of Hong Kong, China, in 2007. His main research areas are analysis and combinatorics, with an emphasis on their applications to coding and information theory.

Brian H. Marcus (M'84-F'07) received the B.A. degree from Pomona College, Claremont, CA, in 1971 and the Ph.D. degree in mathematics from the University of California, Berkeley (UC Berkeley), in 1975.

He held the IBM T.J. Watson Postdoctoral Fellowship in mathematical sciences during 1976-1977. From 1975 to 1985, he was Assistant Professor and then Associate Professor of Mathematics (with tenure) at the University of North
Carolina-Chapel Hill. From 1984 to 2002, he was a Research Staff Member with the IBM Almaden Research Center. He is currently Head and Professor of Mathematics at the University of British Columbia. He has been a Consulting Associate Professor in Electrical Engineering with Stanford University, Stanford, CA, (2000-2003) and Visiting Associate Professor in Mathematics a UC-Berkeley (1986). He is the author of more than 50 research papers in refereed mathematics and engineering journals, as well as a textbook, An Introduction to Symbolic Dynamics and Coding, coauthored with D. Lind (Cambridge, U.K.: Cambridge University Press, 1995). He also holds 10 U.S. patents. His current research interests include constrained coding, error-correction coding, information theory, and symbolic dynamics.

Dr. Marcus was a corecipient of the Leonard G. Abraham Prize Paper Award of the IEEE Communications Society in 1993 and gave an invited plenary lecture at the 1995 International Symposium on Information Theory. He is a member of Phi Beta Kappa, and served as an elected Member of the American Mathematical Society Council (2003-2006). 